\title{
Oozing-Type of Left Ventricular Rupture Treated Under Percutaneous Cardiopulmonary Support Without Surgical Repair
}

\author{
Nobuyuki Masaki, MD; Koh Arakawa, MD; \\ Tadashi Yamagishi, MD; Takanori Tahara, MD; Kouji Miyazaki, MD; \\ Toshio Shibuya, MD; Nobuo Hatori, MD; Hiroshi Yoshizu, MD; \\ Tutomu Tanaka, MD; Fumitaka Ohsuzu, MD
}

\begin{abstract}
A 65-year-old man was admitted to the National Defense Medical College Hospital for acute anterolateral myocardial infarction and cardiogenic shock. Emergency coronary angiography demonstrated occlusion of the proximal left anterior descending artery. Primary percutaneous transluminal coronary angioplasty (PTCA) was successfully performed with the support of intra-aortic balloon pumping (IABP) and medical treatment to stabilize the patient's blood pressure. On the second hospital day, the patient suffered cardiac tamponade. Pericardiocentesis showed bloody fluid and revealed that an oozing-type of left ventricular rupture had occurred after the myocardial infarction. Cardiogenic shock persisted after successful removal of the pericardial effusion. Although the heparinization required during percutaneous cardiopulmonary support (PCPS) can increase pericardial effusion, PCPS was initiated to correct the systemic hypoperfusion; a surgical team was on standby in case massive pericardial effusion resulted, but fortunately that did not occur, and cardiac function recovered. The patient was weaned successfully from PCPS and IABP and has remained in a satisfactory condition for over 1 year. PCPS contributed to the patient's recovery from cardiac shock and may have decreased the effusion from the oozing-type rupture by reducing ventricular wall tension. (Circ J 2002; 66: 769-772)
\end{abstract}

Key Words: Acute myocardial infarction; Cardiogenic shock; Oozing rupture; Percutaneous cardiopulmonary support

$\mathbf{P}$ ercutaneous cardiopulmonary support (PCPS) is effective in maintaining the hemodynamics of a patient with severe cardiogenic shock following acute myocardial infarction (AMI). Of late, PCPS has been used in cases of cardiac rupture, as a bridge to surgical repair 1,2 Cardiac rupture is usually treated by closure of the rupture site, and pericardial effusion or thrombus is an indication for surgical exploration of the pericardium? PCPS in such cases provides hemodynamic stability in patients awaiting surgery. We report a patient in whom PCPS and continuous drainage were used successfully without surgery to treat a left ventricular oozing-type rupture associated with AMI.

\section{Case Report}

A 65-year-old man was admitted to the National Defense Medical College Hospital $3 \mathrm{~h}$ after the onset of severe chest pain. The patient had a 2-month history of effort angina. Upon admission, he was alert, with a systolic blood pressure of $120 \mathrm{mmHg}$ and a heart rate of 100 beats $/ \mathrm{min}$. Electrocardiography (ECG) showed ST elevation in I, aVL and the precordial leads (Fig 1). There were also frequent

(Received May 21, 2001; revised manuscript received August 1, 2001; accepted August 20, 2001)

First Department of Internal Medicine and Second Department of Surgery, National Defense Medical College, Tokorozawa, Japan

Mailing address: Nobuyuki Masaki, MD, First Department of Internal

Medicine and Second Department of Surgery, National Defense

Medical College, 3-2 Namiki, Tokorozawa, Saitama 359-8513, Japan premature ventricular contractions (PVCs). Echocardiography revealed severe hypokinesis of the anteroseptal wall from the base to the apex. AMI was confirmed on the basis of creatine kinase elevation. The PVC frequency was reduced by intravenous administration of mexiletin at an infusion rate of $12.5 \mathrm{mg} / \mathrm{h}$, and the patient was taken to the catheterization laboratory for primary percutaneous transluminal coronary angioplasty (PTCA).

Coronary angiography (CAG) showed $100 \%$ obstruction of the proximal left anterior descending artery (LAD) and $75 \%$ stenosis of the right coronary artery (Fig 2). Intraaortic balloon pumping (IABP) was introduced from the left femoral artery to improve hemodynamic status prior to PTCA because the patient's systolic blood pressure had fallen to $60 \mathrm{mmHg}$ during CAG. Although the LAD was successfully recanalized, no-reflow phenomenon occurred, and $3 \mathrm{mg}$ of isosorbide dinitrate and $100 \mu \mathrm{g}$ of verapamil were administered intracoronary.

Peak creatine kinase was $8,102 \mathrm{IU} / \mathrm{L}$ (10h after onset of chest pain). Peak glutamate oxaloacetate transaminase was $1,022 \mathrm{IU} / \mathrm{L}$, and peak C-reactive protein was $14.7 \mathrm{mg} / \mathrm{ml}$.

On day 2 after admission, the patient complained of chest pain. His systolic blood pressure had dropped again to $60 \mathrm{mmHg}$, and his heart rate increased to 120 beats $/ \mathrm{min}$. Echocardiography revealed pericardial effusion (Fig 3), so an $8 \mathrm{Fr}$ drainage tube was placed in the pericardial space via the subxiphoid approach. We analyzed the pericardial fluid; white blood cell $18,500 / \mathrm{mm}^{3}$, hemoglobin $12.7 \mathrm{~g} / \mathrm{dl}$, hematocrit $36 \%$, platelet $39.5 / \mathrm{mm}^{3}$, total protein $9.0 \mathrm{~g} / \mathrm{dl}$, serum sodium $135 \mathrm{mmol} / \mathrm{L}$, serum potassium $3.3 \mathrm{mmol} / \mathrm{L}$, serum 


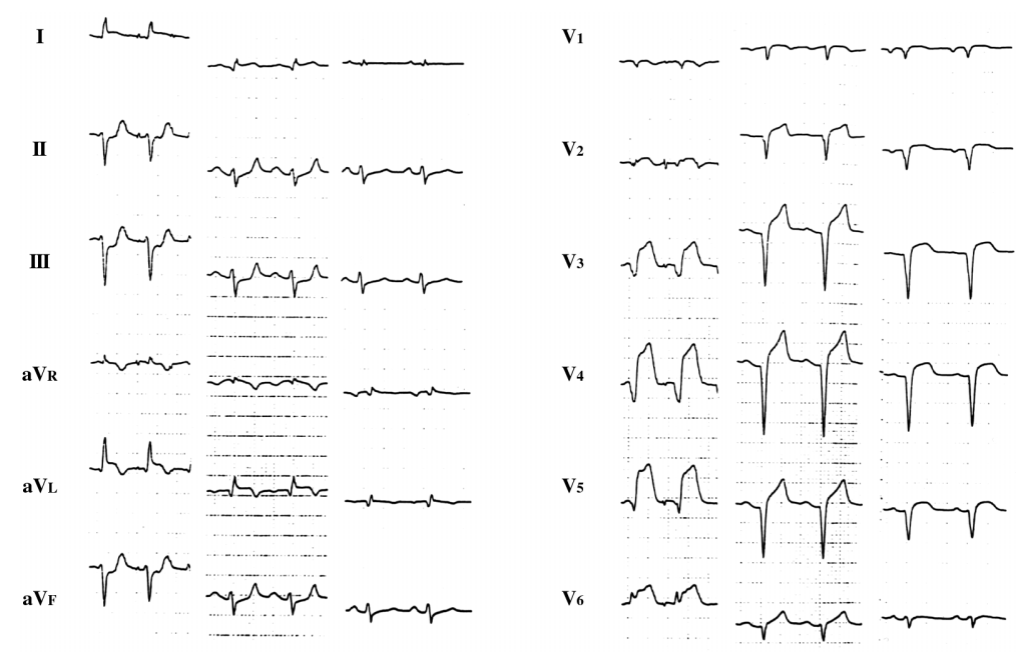

Fig 1. Electrocardiograms on admission (Left), after reperfusion (Middle), and after introduction of PCPS on day 3 (Right). ST-segment elevation is seen in leads $I_{,} a V_{L}$ and $V_{2-6}$, suggestive of a large acute myocardial infarction (Left). A QS pattern is noted in almost all precordial leads.
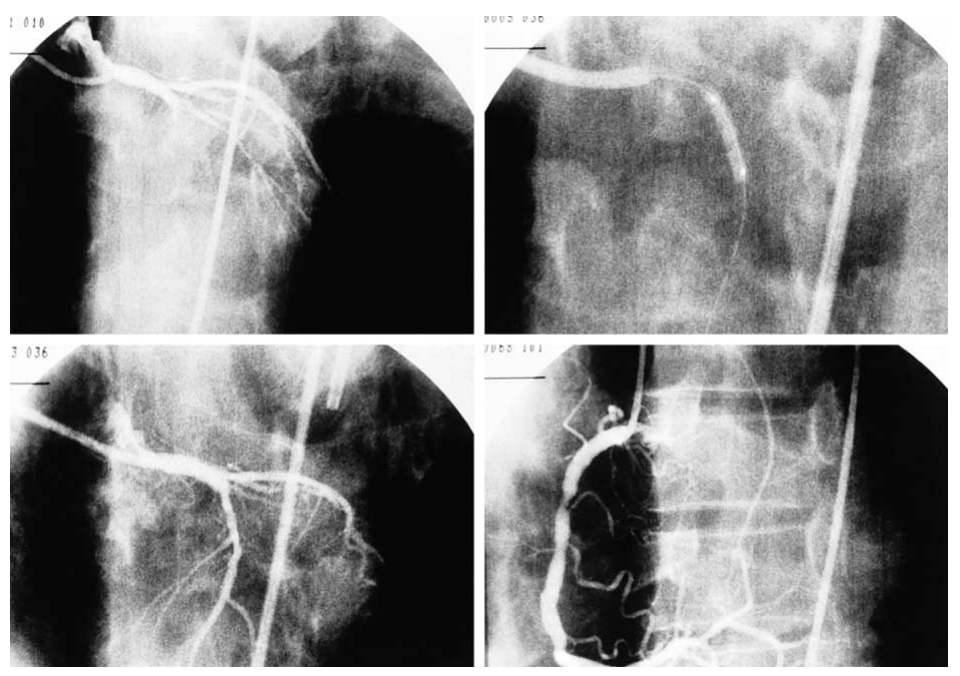

Fig 2. Emergency coronary angiography shows $100 \%$ obstruction of the proximal left anterior descending artery (Upper left). PTCA was performed in this lesion (Upper right). After occurrence of no-reflow phenomenon, intracoronary verapamil and isosorbide dinitrate were administered and delayed flow was established (Lower left). A 75\% stenosis was noted in right coronary artery (Lower right).
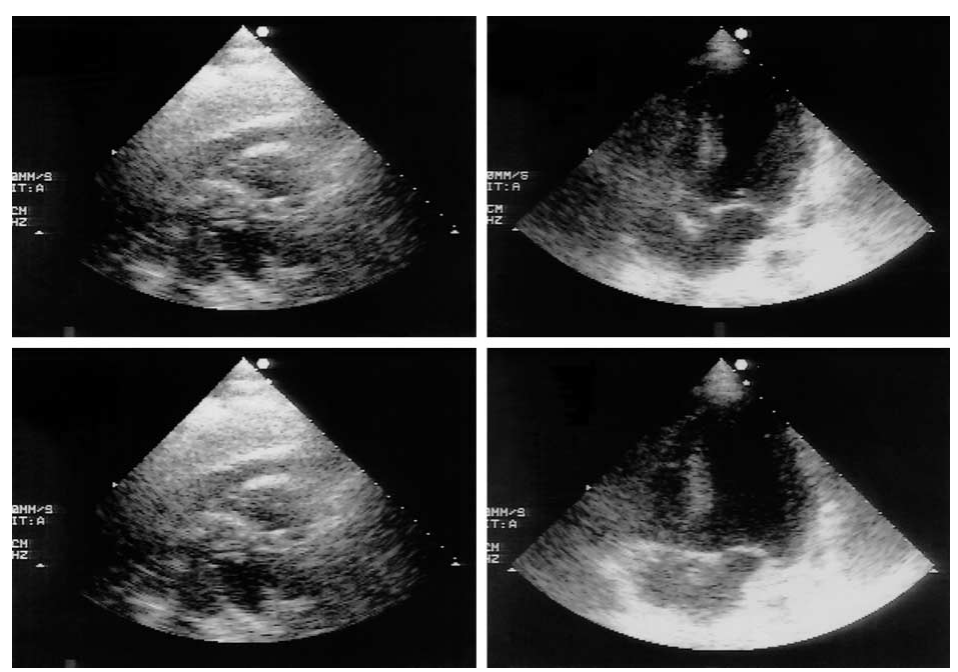

Fig 3. Echocardiography demonstrated an echo-free space 2 days after PTCA; subxiphoidal view (Upper left and Lower left). Echo-free space almost disappeared after urgent pericardiocentesis; 4 chamber view (Upper right and Lower right). Improvement of cardiac shock was not obtained, however.

chloride $97 \mathrm{mmol} / \mathrm{L}, \mathrm{C}$-reactive protein $4.5 \mathrm{mg} / \mathrm{dl}$. These values were almost equal to those of the peripheral blood. Therefore, we suspected a subacute left ventricular rupture. Initially, $125 \mathrm{ml}$ of bloody fluid was removed, and approximately $60 \mathrm{ml}$ was removed over the next $8 \mathrm{~h}$. The patient remained in cardiogenic shock after the first pericardiocentesis, so intubation and sedation were performed for mechanical ventilation. Direct current cardioversion was also performed because the ECG showed ventricular tachycardia during sedation. The pharmacologic treatments and 


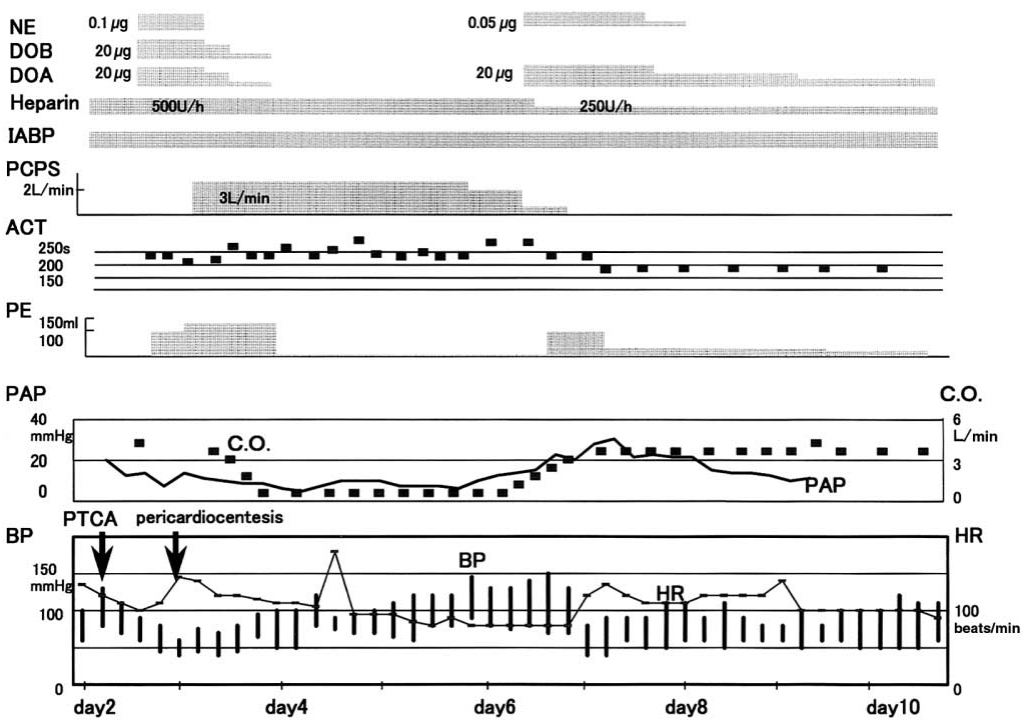

Fig 4. Clinical course of the patient in the intensive care unit. IABP was inserted prior to PTCA on day 2. On the evening of day 2, cardiac tamponade occurred, and bloody fluid was drawn from the pericardial space. PCPS initial flow was $3 \mathrm{~L} / \mathrm{min}$. PCPS was turned off on day 6. PTCA, percutaneous transluminal coronary angioplasty; IABP, intra-aortic balloon pump; PCPS, percutaneous cardiopulmonary support; C.O., cardiac output; PAP, mean pulmonary artery pressure; BP, blood pressure; HR, heart rate; $\mathrm{ACT}$, activated coagulation time; PE, pericardial effusion; NE, norepinephrine; DOA, dopamine hydrochloride; DOB, dobutamine hydrochloride.

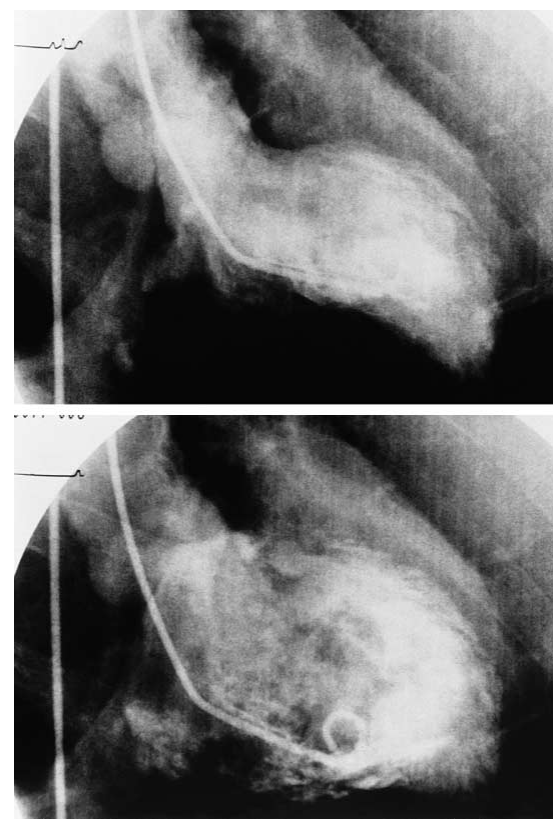

Fig 5. Left ventriculography 1 month after the AMI (Upper). Systole, (Lower) diastole. The anterolateral and apical walls were akinetic.

IABP failed to maintain systolic blood pressure, therefore PCPS was introduced at a controlled flow rate of $3.0 \mathrm{~L} / \mathrm{min}$ (Fig 4) to maintain adequate hemodynamics. We used a Maxima Plus PRFTM Hollow Fiber Oxygenator made with improved plasma-resistant fiber and Carmeda BioActive Surface (Medtronic, Tokyo, Japan) and a heparin-coated circuit. Activated coagulation time was controlled between 200 and $250 \mathrm{~s}$. The patient's condition stabilized at a blood pressure of approximately $80 / 60 \mathrm{mmHg}$ and heart rate of 90 beats/min.

The pericardial discharge diminished gradually to $135 \mathrm{ml}$ on day 4 and $35 \mathrm{ml}$ on days 5 and 6 . Weaning from PCPS was begun on day 6 (Fig 4), after which the pericardial discharge increased temporarily to $107 \mathrm{ml}$. With reduced heparin administration, the pericardial discharge decreased to $30 \mathrm{ml}$ on day $7,16.5 \mathrm{ml}$ on day $8,4.5 \mathrm{ml}$ on day 9 , and
$1.5 \mathrm{ml}$ on day 10. At this point, the patient was hemodynamically stable with a cardiac output of approximately 2.8-3.1 L/min under catecholamine and IABP therapies. The IABP was discontinued on day 11, and catecholamine therapy was successfully terminated on day 19. After day 16 , no fluid could be drawn from the drainage tube. On day 19 , no echo-free space was observed in the pericardium and the pericardial drain was removed. On day 28, CAG demonstrated slow flow and $75 \%$ stenosis in the LAD, and left ventriculography showed akinesis in the anterolateral and apical walls (Fig 5). The patient was discharged and has done well for over 1 year since the AMI.

\section{Discussion}

Cardiac rupture, one of the leading causes of death following AMI, develops in approximately $10 \%$ of patients? There are generally considered to be 2 types, based on clinical course: the blowout type and the oozing type. Prompt surgical closure is the overwhelmingly preferred treatment for cardiac rupture, but although various techniques have been reported, there is little data on operative success. Hospital mortality of patients with subacute rupture is reported at approximately 50\% in some studies:-7 In a study of 33 patients with subacute rupture by Lopez-Sendon et al, immediate operative success was $76 \%$, and long-term survival was $48.5 \%$ ?

There have been few reports of patients with oozing-type cardiac rupture treated without surgical repair-10 ${ }^{8-10}$ Murata et al reported the effectiveness of percutaneous fibrin-glue therapy in lieu of surgery, and Figueras et al treated 19 selected free-wall rupture patients with prolonged rest, blood pressure control therapy, and pericardiocentesis? The authors concluded that medical management could only be recommended in selected patients with very high surgical risk, such as those with a large infarct area or who are over 75 years of age.

PCPS plays an important role in alleviating hypoperfusion of systemic organs and the clinical applications of PCPS include cardiogenic shock for which pharmacologic therapy and IABP are ineffective, refractory ventricular fibrillation, patients who are dead on arrival, respiratory failure, low output syndrome associated with cardiac sur- 
gery, and support for PTCA, especially for proximal lesions of the left coronary artery! ${ }^{1-13}$ PCPS is also used for patients with cardiac rupture after AMI to maintain systemic perfusion prior to surgery, 1,2 but the resuscitation rate for such patients requiring PCPS is unknown because of the small number of patients in whom this technique has been studied ${ }^{14}$

Our experience was with a patient with cardiogenic shock accompanied by cardiac tamponade caused by an oozing-type of left ventricular rupture after a large acute anteroseptal myocardial infarction. Although we performed urgent life-saving pericardiocentesis and placed a drainage tube in the pericardial space, the cardiogenic shock did not improve with medical treatment and IABP support. When the patient's condition further deteriorated after the successful pericardiocentesis, and with a surgical team on standby, we initiated PCPS. Even though the PCPS circuit is coated with heparin, an anticoagulation regimen is required to inhibit clot formation; thus use of PCPS can accelerate hemorrhage into the pericardial space via the rupture site. However, we felt that PCPS was our only treatment option in this case. Fortunately, the pericardial effusion did not increase more than we anticipated during PCPS, and decreased several days after the initiation of PCPS.

We believe that the pericardial effusion did not increase during PCPS because the PCPS reduced left ventricular wall tension by diminishing preload during venous-arterial bypass, thus reducing the flow passing through the left ventricle. Sufficient organ and peripheral tissue perfusion led to the prevention of tachycardia. The absence of inotropic agents may also have contributed to the reduction of ventricular wall tension. PCPS flow may increase afterload, but in this case the patient's systolic blood pressure was well controlled around $100 \mathrm{mmHg}$ and there was no evidence of dilatation of the left atrium or ventricle on echocardiography performed throughout PCPS.

In summary, a patient with an oozing ventricular rupture accompanied by prolonged cardiogenic shock recovered with the aid of mechanical cardiac support and without surgical treatment. Surgical exploration and repair are still the preferred treatments for cardiac rupture, but mechanical cardiac support without surgical repair is one option for certain high-risk patients.

\section{References}

1. Doi K, Sakai M, Ohteki H, Narita Y. Two successful cases of surgical treatment for left ventricular rupture following myocardial infarction: The usefulness of percutaneous cardiopulmonary support system (PCPS). Kyobu Geka 1996; 49: 842-845.

2. Morishita A, Shimakura T, Nonoyama M, Takasaki T, Yoda M. Percutaneous cardiopulmonary support as a bridge to emergency operation -two surviving cases. Jpn Circ J 2000; 64: 528-532.

3. Raitt MH, Kraft CD, Gardner CJ, Pearlman S, Otto CM. Subacute ventricular free wall rupture complicating myocardial infarction. Am Heart J 1993; 126: 946-955.

4. Oliva PB, Hammill SC, Edwards WD. Cardiac rupture, a clinically predictable complication of acute myocardial infarction: Report of 70 cases with clinicopathologic correlations. J Am Coll Cardiol 1993; 22: 720-726.

5. Figueras J, Cortadellas J, Evangelista A, Soler-Soler J. Medical management of selected patients with left ventricular free wall rupture during acute myocardial infarction. J Am Coll Cardiol 1997; 29: $512-518$.

6. Lopez-Sendon J, Gonzalez A, Lopez de Sa E, Coma-Canella I, Roldan I, Dominguez F, et al. Diagnosis of subacute ventricular wall rupture after acute myocardial infarction: Sensitivity and specificity of clinical, hemodynamic and echocardiographic criteria. J Am Coll Cardiol 1992; 19: 1145-1153.

7. Sasaki K, Takami H, Fukuda H, Ohnishi K. Surgical result of left ventricular free wall rupture complicating acute myocardial infarction. Nippon Kyoubu Geka Gakkai Zasshi 1997; 45: 73-78.

8. Murata H, Masuo M, Yoshimoto H, Toyama J, Shimada M, Simamura $\mathrm{Y}$, et al. Oozing type cardiac rupture repaired with percutaneous injection of fibrin-glue into the pericardial space: Case report. Jpn Circ J 2000; 64: 312-315.

9. Mathew TP, Johnston PW, Khan MM, Adgey AA. Long-term survival of a patient with left ventricular free wall rupture without surgical repair. Clin Cardiol 1999; 22: 237-238.

10. Blinc A, Noc M, Pohar B, Cernic N, Horvat M. Subacute rupture of the left ventricular free wall after acute myocardial infarction: Three cases of long-term survival without emergency surgical repair. Chest 1996; 109: 565-567.

11. Sakai M, Ohteki H, Doi K, Masumoto A, Akatsuka H, Hayashida K, et al. Clinical application and indication for percutaneous cardiopulmonary bypass support in cardiopulmonary failure of various etiologies. JJAAM 1996; 7: 345-351.

12. Rees MR, Brown T, Sivananthan UM, Whittaker S, Hick D, Verma SP, et al. Cardiac resuscitation with percutaneous cardiopulmonary support. Lancet 1992; 340: 513-514.

13. Ihno T, Nakagawa T, Furukawa H, Shimizu K, Egi K, Maemura T, et al. Various problems during long-term percutaneous cardiopulmonary support. Artif Organs 1997; 21: 766-771.

14. Matsukawa R, Sakakibara T, Shintani H, Yagura A, Masai T, Hirayama A, et al. Emergency cardiopulmonary bypass support in patients with severe cardiogenic shock after acute myocardial infarction. Heart Vessels 1996; 11: 27-29. 